\title{
Integrated Proteomic and Transcriptomic Analysis of Differential Expression of Chicken Lung Tissue in Response to NDV Infection during Heat Stress
}

\author{
Perot Saelao ${ }^{1,2,3}$, Ying Wang ${ }^{2,3}$, Ganrea Chanthavixay ${ }^{1,2,3}$, Vivian $\mathrm{Yu}^{3}$, Rodrigo A. Gallardo ${ }^{4}$, \\ Jack C. M. Dekkers ${ }^{5}$, Susan J. Lamont ${ }^{5}$, Terra Kelly ${ }^{2,4}$ and Huaijun Zhou ${ }^{2,3, * \mathbb{D}}$ \\ 1 Integrative Genetics and Genomics Graduate Group, University of California, Davis, CA 95616, USA; \\ psaelao@ucdavis.edu (P.S.); kchantha@ucdavis.edu (G.C.) \\ 2 Genomics to Improve Poultry Innovation Lab, University of California, Davis, CA 95616, USA; \\ ucywang@ucdavis.edu (Y.W.); trkelly@ucdavis.edu (T.K.) \\ 3 Department of Animal Science, University of California, Davis, CA 95616, USA; vsyu@ucdavis.edu \\ 4 School of Veterinary Medicine, University of California, Davis, CA 95616, USA; ragallardo@ucdavis.edu \\ 5 Department of Animal Science, Iowa State University, Ames, IA 50011, USA; \\ jdekkers@iastate.edu (J.C.M.D.); sjlamont@iastate.edu (S.J.L.) \\ * Correspondence: hzhou@ucdavis.edu
}

Received: 6 November 2018; Accepted: 21 November 2018; Published: 27 November 2018

\begin{abstract}
Newcastle disease virus (NDV) is a devastating worldwide poultry pathogen with major implications for global food security. In this study, two highly inbred and genetically distinct chicken lines, Fayoumis and Leghorns, were exposed to a lentogenic strain of NDV, while under the effects of heat stress, in order to understand the genetic mechanisms of resistance during high ambient temperatures. Fayoumis, which are relatively more resistant to pathogens than Leghorns, had larger numbers of differentially expressed genes (DEGs) during the early stages of infection when compared to Leghorns and subsequently down-regulated their immune response at the latter stages to return to homeostasis. Leghorns had very few DEGs across all observed time points, with the majority of DEGs involved with metabolic and glucose-related functions. Proteomic analysis corroborates findings made within Leghorns, while also identifying interesting candidate genes missed by expression profiling. Poor correlation between changes observed in the proteomic and transcriptomic datasets highlights the potential importance of integrative approaches to understand the mechanisms of disease response. Overall, this study provides novel insights into global protein and expression profiles of these two genetic lines, and provides potential genetic targets involved with NDV resistance during heat stress in poultry.
\end{abstract}

Keywords: chicken; Newcastle disease virus; RNA-seq; proteomics

\section{Introduction}

Newcastle disease virus (NDV) has become one of the most important avian diseases impacting poultry production and trade worldwide. NDV is a highly contagious viral disease, capable of spreading rapidly among populations of birds and decimating poultry flocks [1]. The World Organization for Animal Health includes virulent forms of NDV as a specific hazard due to the impact that NDV outbreaks have on international trade and animal welfare [2]. Proper vaccination, along with effective use of biosecurity and biosafety measures on poultry farms, have been largely successful in preventing infections by NDV. However, understanding host genetic diversity and heterogeneity in the immune response can aid in supporting these measures and inform upon the genetic factors involved in effectively responding to infection. 
Changing climate conditions is an additional factor that may impact the host's resistance and response to disease. Extreme weather events have been found to create more frequent [3] and intense [4] disease outbreaks. Studies on seasonality, and the effects of temperature on Newcastle disease outbreaks have noted higher incidences during the hot and dry, and hot and humid seasons [5,6]. Additionally, investigations into the specific effects of heat stress on the immune response in chickens have shown immunosuppressing effects through reduced lymphoid organ weights $[7,8]$, lower circulating antibodies [9], and altered systemic humoral response [10].

Several studies have investigated the role that host genetics plays in explaining differences in response to NDV without heat treatment [11-13] in chickens. These studies found Fayoumi chickens, native to Egypt and imported to the U.S. in 1954, to be relatively more resistant to the La Sota strain of NDV, with significantly lower viral load and higher circulating anti-NDV antibodies, than Leghorn chickens [12,14]. Furthermore, Fayoumis has demonstrated higher resistance to other viral pathogens such as avian influenza [15] and Marek's disease [16]. Transcriptome profiling of the two genetic lines during NDV infection has identified many candidate genes and signaling pathways potentially regulating the tissue-specific response to NDV infection [11-13]. Specifically, the mucosal surface of the airways in chickens is one of the main sites targeted for infection and entry by respiratory pathogens such as NDV. The innate and adaptive immune response of tissues in the respiratory tract, including the lungs, are of critical importance to limiting the systemic spread of the virus in the bird.

Large-scale analysis of the proteome can accurately determine the relative abundance of proteins within tissues. Proteomics offers the opportunity to comprehensively investigate the changes in signaling pathways and biological processes that would otherwise be missed by transcriptome sequencing. In addition, quantitative proteomics and the integrative analysis of the transcriptome can provide corroborative evidence for high priority targets to aid in the discovery of unique mechanisms. The objective of this study was to identify candidate genes and proteins involved with the chicken response to NDV infection during heat stress using an integration of proteomic and transcriptomic approaches. We believe this integrated approach will identify novel biomarkers and provide insight into the differences in response to NDV infection during high ambient temperatures between Fayoumi and Leghorn chicken lines.

\section{Materials and Methods}

\subsection{Experimental Populations and Design}

The experimental design of this study has been previously described by Wang et. al. 2018 [17]. Briefly, Fayoumi (M15.2) and Leghorn (GHs 6) inbred chicken lines from Iowa State University (Ames, IA, USA) were used in this study. On the day of hatching, 56 Fayoumi and 55 Leghorn chicks were transported from Iowa State University to the University of California, Davis. Upon arrival, the chicks were housed in temperature- and humidity-controlled chambers in a biosafety level 2 animal facility. Twenty-five individuals from each genetic line were randomly selected and housed in a separate chamber to be used as the control group. From day 1 to day 13 both groups were reared at $29.4{ }^{\circ} \mathrm{C}$ and $60 \%$ humidity. At 14 days of age, the experimental group was exposed to $38^{\circ} \mathrm{C}$ for $4 \mathrm{~h}$, then decreased to $35^{\circ} \mathrm{C}$ and maintained at this temperature until conclusion of the trial. The control group was maintained at $29.4{ }^{\circ} \mathrm{C}$ and gradually decreased to $25^{\circ} \mathrm{C}$. On day 21 , the heat-treated

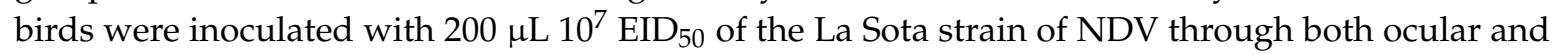
nasal passages. The control group was mock inoculated with $200 \mu \mathrm{L}$ of $1 \mathrm{X}$ phosphate-buffered saline (PBS). At 2, 6, and 10 days post-infection (dpi), four birds per treatment group per genetic line were randomly selected and euthanized with $\mathrm{CO}_{2}$. Lung tissue was harvested and quickly placed into RNAlater (ThermoFisher, Waltham, MA USA Cat\#AM7024) and kept at $-80^{\circ} \mathrm{C}$. Additional lung tissue samples were collected at 2 and $6 \mathrm{dpi}$ and snap frozen in liquid nitrogen and then stored at $-80^{\circ} \mathrm{C}$ until protein extraction. Protein extraction, protein assay, and the in-solution trypsin digestion were performed as reported by Kultz et. al. [18]. The experiment's procedures were performed according 
to the guidelines approved by the Institutional Animal Care and Use Committee at the University of California, Davis (IACUC \#17853).

\subsection{RNA Isolation and Library Construction}

Total RNA was extracted from the lung of four individuals per treatment and genetic line for each of the three time points. The lung tissue was homogenized in ice cold TRIzol (ThermoFisher, Waltham, MA USA Cat\#15596026) and processed using a standard phenol:chloroform method and precipitated in $100 \%$ ethanol. The RNA pellet was then dissolved into water and treated with DNase I (ThermoFisher, Cat\#EN0521). Strand-specific RNA library preparation was prepared using the NEBNext Ultra Directional RNA Library Prep Kit for Illumina (NEB, Ipswich, MA USA Cat\#E7420S) according to the manufacturer's instructions. Library validation and quantification was conducted using the Agilent Bioanalyzer High Sensitivity Kit (Agilent, Santa Clara, CA USA Cat\#5067-4626) and Qubit dsDNA HS Assay kit (ThermoFisher, Cat\#Q32854). The 100 base pair, paired-end sequencing was performed on the Illumina HiSeq2500 system with a minimum sequencing depth of 30 million reads per sample. Sequence data have been submitted through the Sequence Read Archive under accession number: SRP155740.

\subsection{Protein Identification and Quantification}

Protein identification and quantification was performed as previously described by Kultz et. al. (2016) [19]. Briefly, peptides from each sample were separated by reverse phase liquid chromatography and then analyzed by an ImpactHD UHR-QTOF mass spectrometer (Bruker Daltonics, Bremen, Germany). Peak lists were imported into PEAKS 7.5 (BSI, Waterloo, Canada) and PEAKS, Mascot 2.2 (Matrix Science, London, UK) and X!Tandem Cycle [19] search engines used to identify proteins from the MS/MS spectra. Search results from all three methods were combined in Scaffold 4.4 (Proteome Software Inc., Portland, OR, USA) with peptide false discovery rate (FDR) $<0.1$, protein FDR $<0.1$, and peptides per protein $\geq 2$. Label-free quantitative profiling of peptide intensities was performed in PEAKS 7.5, and all identification and profiling data were made publicly available at the CAMP proteome at the University of California, Davis (Davis, CA USA, AC\# CAMPDDA00051). Pathway analysis of differentially abundant proteins, DAPs, identified at each timepoint between conditions was done using STRING [20]. The correlation between the $\log _{2}$ change in protein abundance and $\log _{2}$ fold change in gene expression was calculated on 585 protein-transcript pairs. Spearman's correlation coefficient and significance value was determined using R's cor.test function [21] for all timepoints.

\subsection{Data Analysis}

Four major factors were included for analysis: condition (treated, non-treated), line (Leghorn, Fayoumi), sex (male, female), and time point (2, 6, and $10 \mathrm{dpi}$ ). Data at each time point consisted of 16 individuals, four per treatment and genetic line. Raw reads from RNA-seq were trimmed using FastQC [22] to remove duplicates, reads with base quality scores $<30$, and adapter contamination. These reads were then aligned using STAR [23] to the galGal5 reference genome and Ensembl annotation using default settings. Gene counts were calculated using HTSeq [24] and differential gene analysis was done using edgeR [25]. The statistical model design included the effects of line, condition, sex, and time point. In addition, in order to identify genes that were differentially expressed between genetic lines in response to treatment and, therefore, potentially associated with disease resistance to NDV, the interaction between condition and genetic line was included. Genes were identified as differentially expressed if they had an FDR $<0.05$, and an average transcript count $>10$. Pathway analysis using the DEGs of within line contrasts was performed using Qiagen's Ingenuity Pathway Analysis software [26]. Z-score cutoff of $|z|>1$ identified significantly up- or down-regulated pathways [26]. 


\section{Results}

\subsection{Differential Gene Expression from within-Line Comparisons}

Differential gene expression analysis between treated and non-treated individuals was performed within both Fayoumi and Leghorn chickens at 2, 6, and 10 dpi to explore changes in gene expression induced by NDV infection and heat stress. Comparisons between treated and non-treated Fayoumi chickens at 2 dpi identified 122 differentially expressed genes (DEGs) that were up-regulated and 58 DEGs that were down-regulated (Table 1). The number of DEGs subsequently decreased to 26 up-regulated and 27 down-regulated DEGs at the $6 \mathrm{dpi}$ timepoint. The largest number of DEGs identified within line was at 10 dpi with 173 up-regulated and 358 down-regulated DEGs. For Leghorns, 35 DEGs were up-regulated while only two DEGs were down-regulated at 2 dpi. Principal component analysis to identify sample clustering for the transcriptomic and proteomic data can be found in the Supplementary Figures (Figures S1 and S2). For both the transcriptomic (Figure S1) and proteomic (Figure S2) datasets, the samples appeared to cluster by genetic line and then by dpi. In both datasets, the samples did not appear to cluster by treatment state across all three time points. Very few genes were differentially expressed at 6 dpi with only six DEGs up-regulated and one DEG that was down-regulated. At 10 dpi, Leghorns had only 10 and five DEGs up-regulated and down-regulated, respectively.

Table 1. Number of differentially expressed genes (DEGs) and differentially abundant proteins (DAPs) in Fayoumis and Leghorns at each timepoint with a false discovery rate (FDR) $<0.05$.

\begin{tabular}{cccccc}
\hline & & \multicolumn{2}{c}{ Differentially Expressed Genes } & \multicolumn{2}{c}{ Differentially Abundant Proteins } \\
\hline Line & Days Post-Infection & Up-Regulated & Down-Regulated & Up-Regulated & Down-Regulated \\
\hline Fayoumi & 2 & 122 & 58 & 0 & 6 \\
Leghorn & 2 & 35 & 2 & 5 & 5 \\
Fayoumi & 6 & 26 & 27 & 4 & 37 \\
Leghorn & 6 & 6 & 358 & & \\
Fayoumi & 10 & 173 & 5 & & \\
Leghorn & 10 & 10 & & \\
\hline
\end{tabular}

\subsection{Pathway Analysis of Differentially Expressed Genes}

Ingenuity pathway analysis (IPA) of the DEGs identified in the Fayoumi and Leghorn within line comparisons revealed canonical pathways unique to each genetic line (Figure 1A-F). Fayoumi had eight and 19 significant pathways enriched at 2 and 6 dpi, respectively. Notable pathways include the phagosome maturation (2 dpi), serotonin receptor signaling (2 dpi), IL-9 signaling (6 dpi), ERK/MAPK timepoint (6 dpi), and autophagy signaling (6 dpi) pathways. Pathway enrichment at $10 \mathrm{dpi}$ identified 47 significantly enriched pathways, with 28 of these pathways with a predicted negative Z-score suggesting down-regulation and one pathway with a predicted positive Z-score (Table 2). These down-regulated pathways include IL-8 signaling, B cell receptor signaling, and NF-kappaB signaling which are prominent pathways involved in the immune response. Very few pathways were enriched among the Leghorn DEGs across all three timepoints. At $2 \mathrm{dpi}$, only six pathways were significantly enriched, and of these pathways the majority were involved with glycogen metabolism including glycogen degradation II, glycogen degradation III, and glucose and glucose-1-phosphate degradation pathways. Similarly, these three glycogen and glucose degradation pathways were again among the seven enriched pathways at the $6 \mathrm{dpi}$ timepoint for Leghorns. Analysis at the $10 \mathrm{dpi}$ timepoint identified only four significantly enriched pathways. 

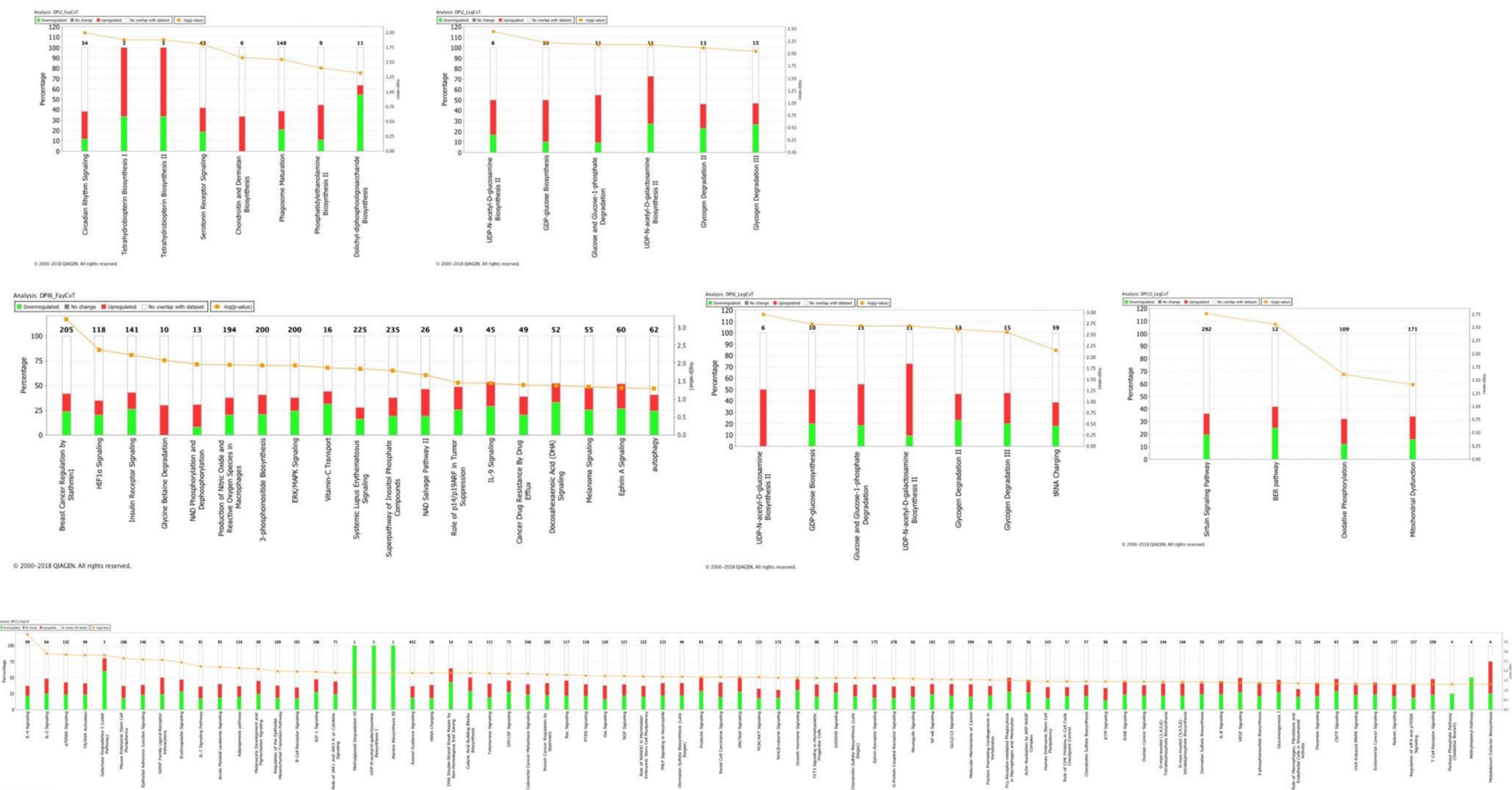

Figure 1. Significantly enriched pathways identified through the Ingenuity Pathway Analysis among the differentially expressed genes by timepoint and genetic line. (A) Fayoumi at 2 days post-infection (dpi), (B) Leghorn at 2 dpi, (C) Fayoumi at 6 dpi, (D) Leghorn at 6 dpi, (E) Fayoumi at 10 dpi, and (F) Leghorn at 10 dpi. The number of genes in each pathway is shown in black, up-regulated (red) and down-regulated genes (green) within the pathway, and the significance value calculated as the $-\log (p$-value $)$ is depicted as an orange line. 
Table 2. Top canonical pathways significantly enriched between treated and non-treated Fayoumis at $10 \mathrm{dpi}$. Activated pathways are Z-score $>0$ and inhibited pathways are Z-score $<0$. NA designates pathways without a predicted activity score.

\begin{tabular}{|c|c|}
\hline Canonical Pathway & Z-Score \\
\hline ERK/MAPK Signaling & -2.24 \\
\hline Thrombin Signaling & -2.24 \\
\hline mTOR Signaling & -2.00 \\
\hline Fcy Receptor-mediated Phagocytosis in Macrophages and Monocytes & -2.00 \\
\hline p70S6K Signaling & -1.89 \\
\hline B Cell Receptor Signaling & -1.89 \\
\hline Colorectal Cancer Metastasis Signaling & -1.89 \\
\hline Mouse Embryonic Stem Cell Pluripotency & -1.63 \\
\hline Rac Signaling & -1.34 \\
\hline Ga12/13 Signaling & -1.34 \\
\hline IL-8 Signaling & -1.34 \\
\hline NF-kappaB Signaling & -1.34 \\
\hline Regulation of eIF4 and p70S6K Signaling & -1.34 \\
\hline NGF Signaling & -1.34 \\
\hline Telomerase Signaling & -1.34 \\
\hline Acute Myeloid Leukemia Signaling & -1.34 \\
\hline Fc Epsilon RI Signaling & -1.00 \\
\hline fMLP Signaling in Neutrophils & -1.00 \\
\hline ErbB Signaling & -1.00 \\
\hline Growth Hormone Signaling & -1.00 \\
\hline EIF2 Signaling & -1.00 \\
\hline FLT3 Signaling in Hematopoietic Progenitor Cells & -1.00 \\
\hline Melanocyte Development and Pigmentation Signaling & -1.00 \\
\hline Renal Cell Carcinoma Signaling & -1.00 \\
\hline Neuregulin Signaling & -1.00 \\
\hline Insulin Receptor Signaling & -1.00 \\
\hline GM-CSF Signaling & -1.00 \\
\hline Sirtuin Signaling Pathway & -0.45 \\
\hline Prolactin Signaling & 0.00 \\
\hline PTEN Signaling & 0.00 \\
\hline Neuroinflammation Signaling Pathway & 0.45 \\
\hline AMPK Signaling & NA \\
\hline G Beta Gamma Signaling & NA \\
\hline FcyRIIB Signaling in B Lymphocytes & NA \\
\hline Renin-Angiotensin Signaling & NA \\
\hline Role of NFAT in Cardiac Hypertrophy & NA \\
\hline eNOS Signaling & NA \\
\hline Huntington's Disease Signaling & NA \\
\hline GP6 Signaling Pathway & NA \\
\hline ErbB4 Signaling & NA \\
\hline Lymphotoxin B Receptor Signaling & NA \\
\hline PAK Signaling & NA \\
\hline CD40 Signaling & NA \\
\hline Production of Nitric Oxide and Reactive Oxygen Species in Macrophages & NA \\
\hline alpha-Adrenergic Signaling & NA \\
\hline P2Y Purigenic Receptor Signaling Pathway & NA \\
\hline Amyotrophic Lateral Sclerosis Signaling & NA \\
\hline
\end{tabular}

\subsection{Quantification of Protein Levels and Differential Protein Abundance}

Proteins from lung tissue were extracted and quantified from the same birds used for transcriptome analysis at 2 and 6 dpi to identify significantly over or under abundant proteins expressed during the early immune response. The primary goal for protein quantification was to investigate the early stages of infection, and the early proteomic response observed within these timepoints. The 10 dpi timepoint was not investigated for protein quantification due to sample 
limitations and central focus of the early immune response. Comparing treated to non-treated Fayoumis, we found six and 13 DAPs at 2 and 6 dpi, respectively (Table 1). Differentially abundant proteins are proteins that have been identified as differentially abundant in either the treated or non-treated groups. The same comparisons made within Leghorns revealed 10 DAPs at 2 dpi. The largest number of DAPs was identified at 6 dpi with 99 DAPs found to be differentially abundant between treated and non-treated birds. No significant pathways were identified within Fayoumis at either timepoint and for Leghorn at 2 dpi using STRING. However, KEGG pathway enrichment analysis found 10 significant pathways enriched among the 99 DAPs at 6 dpi in Leghorns (Figure 2). Similar metabolic-related pathways identified from the differential gene expression analysis in Leghorns were found among the pathways enriched in the protein dataset.

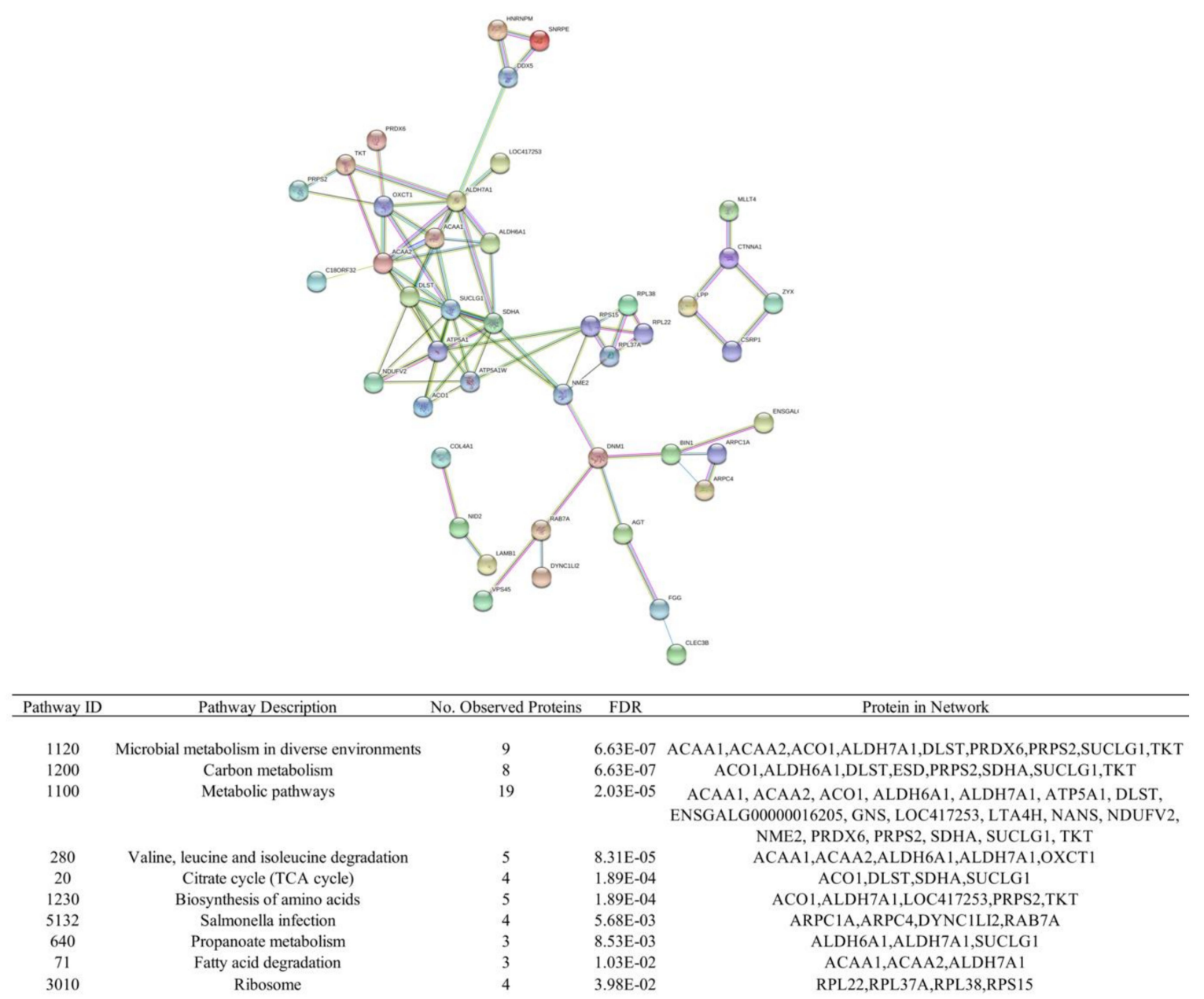

Figure 2. STRING network analysis on DAPs between treated and non-treated Leghorns at 6 dpi. Nodes depict protein-protein interactions. Figure table lists the significantly enriched pathways and their corresponding differentially abundant protein within these pathways.

\subsection{Correlation of Change in Protein Abundance and Differential Gene Expression}

The strength of the relationship between the change in protein abundance and gene expression changes due to NDV infection and heat treatment were determined for each line and comparison (Table 3). A total of 585 protein-transcript pairs were identified across 2 and 6 dpi in order to observe protein and gene expression correlations (Figure 3). Correlation between the change in protein abundance and gene expression differences due to treatment at both time points in Leghorns and at 2 dpi in Fayoumis were weakly negatively correlated by Pearson correlation analysis. Fayoumi at 6 dpi had weakly positive correlation, however, none of the correlations at any timepoint within 
either genetic line was significant. Additional comparisons between the normalized read counts and relative protein abundances also failed to find significant association between the two datasets across all comparisons except for non-treated samples at 6 dpi in the Fayoumi line (Additional File 1).

Table 3. Correlation coefficients and $p$-value statistics calculated between the $\log _{2}$ (fold change) in gene expression and the $\log _{2}$ (fold change) in protein abundance between treated and non-treated birds at each timepoint.

\begin{tabular}{c|ccc}
\hline \multicolumn{1}{c}{ Line } & Days Post-Infection & $\boldsymbol{r}$ & $\boldsymbol{p}$-Value \\
\hline Fayoumi & 2 & -0.0072 & 0.86 \\
Leghorn & 2 & -0.053 & 0.22 \\
Fayoumi & 6 & 0.051 & 0.22 \\
Leghorn & 6 & -0.018 & 0.65 \\
\hline
\end{tabular}

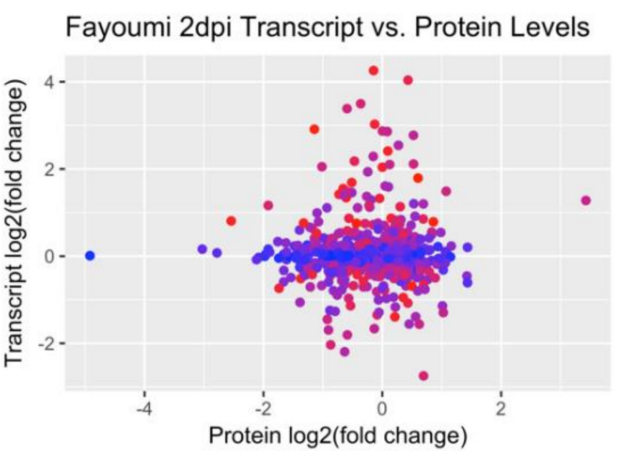

Leghorn 2dpi Transcript vs. Protein Levels

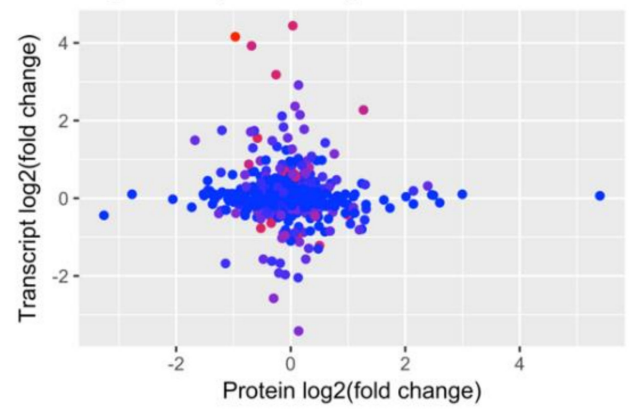

Fayoumi 6dpi Transcript vs. Protein Levels

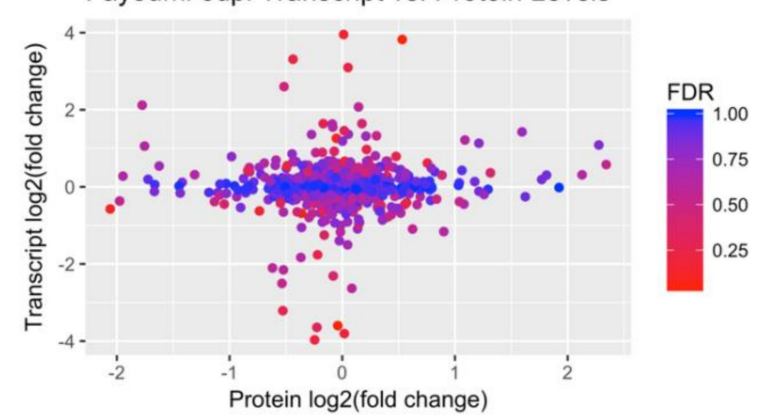

Leghorn 6dpi Transcript vs. Protein Levels

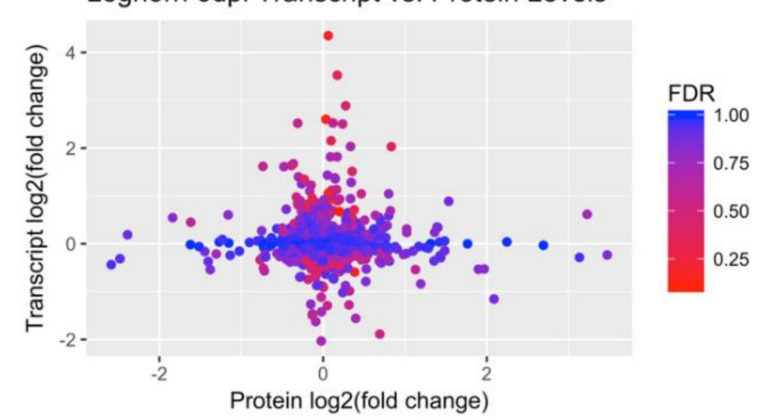

Figure 3. Correlation between the $\log _{2}$ (fold change) in gene expression and the $\log _{2}$ (fold change) in protein abundance between treated and non-treated birds at each timepoint. Intensity of each point represents the q-score of the gene.

\section{Discussion}

To our knowledge, this study is the first to simultaneously address the molecular events that occur during NDV infection and heat stress using global proteomic and transcriptomic profiling techniques in chickens. Our study investigated the dynamic protein and gene expression changes induced in the lung by NDV at 2, 6, and 10 dpi to understand the host response during heat stress. Differential gene expression analysis between disease states found interesting differences in response patterns when comparing the relatively resistant Fayoumis to the more disease susceptible Leghorns. In the Fayoumi comparisons, there was a large number of DEGs identified at 2 dpi with the majority of the DEGs (122) significantly up-regulated. Notable immune-related genes include IL17REL, which is involved in cytokine-mediated signaling, and NOX4, $P R D X 1, R A B 7 B$, which are part of the phagosome maturation pathway. Interestingly, very few genes were differentially expressed at $6 \mathrm{dpi}$, however, results from our group show a similar result in the Harderian gland tissue of the same individuals [14]. The $10 \mathrm{dpi}$ timepoints had many DEGs identified but most of the genes were significantly down-regulated (358). This could be an indication of a resolution of the immune response with genes involved with immunity 
being down-regulated for the maintenance of tissue homeostasis in Fayoumies. Similar studies in chicken Harderian gland, lung, and trachea have also observed a down-regulation of DEGs at 10 dpi providing further indication that the Fayoumis may have resolved the infection and are returning to homeostasis [11,12,14]. Compared to Fayoumis, Leghorns had very few DEGs identified across all three timepoints in this study. The low number of DEGs may suggest that the lungs of Leghorn birds are not responding as effectively to NDV infection during heat stress. One gene that is of particular interest is $P G M 3$, which was significantly up-regulated at both the 2 and 6 dpi timepoints in Leghorns. $P G M 3$ encodes for a protein that mediates glycogen formation and utilization [27]. Studies on PGM3 mutants demonstrate immune deficiency [28], decreased cytokine production [29], and increased susceptibility to avian influenza [30].

Pathways enriched among the DEGs identified provide insight into the potential mechanisms and gene groups utilized in response to NDV infection during heat stress. At 2 dpi, Fayoumi had significant enrichment in two immune-related pathways, serotonin receptor signaling and phagosome maturation. Serotonin receptor signaling is of particular interest due to its role in hormonal regulation of immune function and high expression amongst immune cells with several immunoregulatory functions such as cytokine secretion and T-cell activation [31]. Differentially expressed genes from 6 dpi had a similar trend in enrichment of immune-related pathways (IL-9 signaling, ERK/MAPK, autophagy signaling) amongst the small number of significant pathways. These pathways may be critically important for the enhanced disease resistance observed in Fayoumis. In addition, IPA predicted down-regulation of enriched pathways at $10 \mathrm{dpi}$. However, the neuroinflammation signaling pathway was weakly activated at the 10 dpi timepoint within Fayoumis. Velogenic and mesogenic strains of NDV often localize within the central nervous system (CNS) where it can result in systemic disease and rapid mortality [32]. Neuroinflammation can drive the activation of many pro-apoptotic pathways in an attempt to alleviate infection but may also result in increased pathology of neuro diseases [32]. While the activation of this pathway was limited (z-score $=0.45)$, it is worth noting the potential benefits of the neuroinflammation response observed in the putatively NDV resistant Fayoumis. Fayoumis' down-regulation and modulation of the immune system to return to homeostasic levels can be viewed as characteristic of an efficient immune response at the early stage of infection.

While Fayoumis appears to be activating pathways involved with the immune and antiviral response at 2 and $6 \mathrm{dpi}$, Leghorns were primarily enriched for metabolic-related pathways such as glycogen degradation II and III, and glucose and glucose-1-phosphate degradation at these timepoints. Leghorns appear to be responding to the effects of infection-induced hyperglycemia at both the 2 and 6 dpi timepoints. During infection, it is common to observe increased glucose utilization during increased periods of fasting. Failure of peripheral glucose clearance can lead to hyperglycemia and result in the dysregulation of the immune response [33]. The induction of a fasting state due to both infection and heat stress, and a dysregulation of the glucose-related processes in Leghorn, could potentially explain the poor performance of their immune response.

Despite the similarities in pathways and genes enriched in the proteome and transcriptome data, significant correlation was not observed between the two datasets. Many studies have shown evidence that protein and mRNA levels tend to have poor correlation, especially amongst diverse cell types $[34,35]$. This may be due to various post-transcriptional or post-translational events that would be difficult to observe within our datasets. In addition, the in vivo dynamics in our dataset may not necessarily recapitulate the in vitro systems typically reported when comparing the two methodologies. However, our study identified unique DAPs between treated and non-treated groups that were not identified in the transcriptome analysis. Of the DAPs identified in Fayoumi, ATOX1, SFTPA1 and LL are of interest due to their prominent roles in the innate immune system and defense against respiratory pathogens. Yeast two-hybrid screens found ATOX1 to interact with partners in the TRIM family of proteins related to the immune response [36] and inhibition of viral growth [37]. LL and SFTPA1 are surfactant-related proteins predominantly found within the lung and are involved in the defense against respiratory pathogen and modulation of ligand C-type lectin-related-b (Clr-b) proteins [38]. 
A study in cell lines and mice observed that decreases in surfactant proteins leading to a decrease in clr-b, increase natural killer (NK) cells' ability to sense virus-infected cells and increase NK cell response [39]. However, within Leghorns, proteomic analysis appeared to capture similar observations of increased metabolic and glucose-related processes seen within RNA expression data. COL1A2 was a protein differentially abundant at 2 dpi between Leghorn treatment groups and family collagen-related genes have been observed to be differentially expressed in studies of NDV infection in the lung and trachea [11,12]. At the 6 dpi timepoint, there was significant enrichment of KEGG pathways which include: metabolic, carbon metabolism, citrate cycle (TCA cycle), and fatty acid degradation. It appears that Leghorns were having a more profound alteration of lung metabolism due to infection that may be causing a dysregulation of their immune response. In vivo studies have observed the magnitude of infection and inflammation which result in profound activation of the glucoregulatory systems [40]. The infection status within Leghorns may be substantial enough to shift the body to a state of metabolic inflexibility, typically observed during high levels of inflammatory stress.

In summary, comparisons within Fayoumis and Leghorns using protein and gene expression profiling identified unique significant pathways and candidate genes involved in the lung's response to NDV infection during heat stress. The integration of these two methods allowed for global profiling of the birds' responses and provided corroborative evidence for observations witnessed in the gene expression and protein studies. Additionally, our study identified potentially important sets of genes and proteins which would have been missed through the independent use of either method. The potential roles and underlying molecular mechanisms involved in NDV response during heat treatment require further investigation into the candidate genes and signaling pathways identified.

Supplementary Materials: The following are available online at http:/ /www.mdpi.com/2073-4425/9/12/579/s1, Figure S1: Principle component analysis of sample clustering of all RNA-Seq samples. Samples appear to cluster primarily due to the genetic line rather than treatment state, Figure S2: Principal component analysis demonstrating the clustering of proteomic samples at A) 2 dpi and B) 6 dpi.

Author Contributions: P.S.: Collected samples, isolated RNA, constructed libraries, processed and analyzed data, wrote manuscript; Y.W.: Collected samples, isolated viral RNA, performed qRT-PCR, reviewed and edited manuscript; G.C., V.Y.: Collected samples, isolated RNA and protein samples, reviewed and edited manuscript; R.A.G.: Experimental design, viral isolate preparation, collected samples, reviewed and edited manuscript. J.C.M.D., S.J.L., T.K.: Experimental design, reviewed and edited paper; H.Z.: Experimental design, reviewed and edited manuscript, oversaw data analysis and sample collection.

Funding: This study was funded by the US Agency for International Development Feed the Future Innovation Lab for Genomics to Improve Poultry AID-OAA-A-13-00080. Partial support was provided by the United States Department of Agriculture, National Institute of Food and Agriculture, Multistate Research Project NRSP8, NC1170, NC1180 and NE1334 (HZ) and the California Agricultural Experimental Station (HZ).

Acknowledgments: The authors thank the members of the Zhou and Gallardo lab for their assistance in collecting and preparing samples from the live bird trial.

Conflicts of Interest: The authors declare that they have no competing interests.

\section{References}

1. MacLachlan, N.J.; Dubovi, E. Paramyxoviridae and Pneumoviridae. In Fenner's Veterinary Virology; Elsevier: New York, NY, USA, 2017; pp. 327-356.

2. OIE-Listed diseases, Infections and Infestations in Force in 2018. 2018. Available online: http: / /www.oie. int/en/animal-health-in-the-world/oie-listed-diseases-2018/ (accessed on 12 July 2018).

3. Epstein, P.R. Climate change and emerging infectious diseases. Microbes Infect. 2001, 8, 747-754. [CrossRef]

4. Wu, X.; Lu, Y.; Zhou, S.; Chen, L.; Xu, B. Impact of climate change on human infectious diseases: Empirical evidence and human adaptation. Environ. Int. 2016, 86, 14-23. [CrossRef] [PubMed]

5. George, M.M. Epidemiology of Newcastle disease and the need to vaccinate local chickens in Uganda. In Proceedings of the International Workshop on Oil Palm Germplasm and Utilization, Kuala Lumpur, Malaysia, 12-13 September 1991; pp. 155-158.

6. Mishra, U. Present status of poultry in Nepal. In Proceedings of the 7th Tanzania Veterinary Association Scientific Conference, Arusha, Tanzania, 5 December 1989; pp. 403-407. 
7. Ghazi, S.; Habibian, M.; Moeini, M.M.; Abdolmohammadi, A.R. Effects of different levels of organic and inorganic chromium on growth performance and immunocompetence of broilers under heat stress. Biol. Trace Elem. Res. 2012, 146, 309-317. [CrossRef] [PubMed]

8. Quinteiro-Filho, W.M.; Ribeiro, A.; Ferraz-de-Paula, V.; Pinheiro, M.L.; Sakai, M.; Sa, L.R.M.; Ferreira, A.J.P.; Palermo-Neto, J. Heat stress impairs performance parameters, induces intestinal injury, and decreases macrophage activity in broiler chickens. Poultry Sci. 2010, 89, 1905-1914. [CrossRef] [PubMed]

9. Bartlett, J.R.; Smith, M.O.; Yan, Q.; Li, L. Effects of different levels of selenium on growth performance and immunocompetence of broilers under heat stress. Poultry Sci. 2003, 82, 1580-1588. [CrossRef] [PubMed]

10. Bozkurt, M.; Kucukyilmaz, K.; Catli, A.U.; Cinar, M.; Bintas, E.; Coven, F. Performance, egg quality, and immune response of laying hens fed diets supplemented with mannan-oligosaccharide or an essential oil mixture under moderate and hot environmental conditions. Poultry Sci. 2012, 91, 1379-1386. [CrossRef] [PubMed]

11. Deist, M.S.; Gallardo, R.A.; Bunn, D.A.; Dekkers, J.C.M.; Zhou, H.; Lamont, S.J. Resistant and susceptible chicken lines show distinctive responses to Newcastle disease virus infection in the lung transcriptome. BMC Genom. 2017, 18, 1-15. [CrossRef] [PubMed]

12. Deist, M.S.; Gallardo, R.A.; Bunn, D.A.; Kelly, T.R.; Dekkers, J.C.M.; Zhou, H.; Lamont, S.J. Novel mechanisms revealed in the trachea transcriptome of resistant and susceptible chicken lines following infection with Newcastle disease virus. Clin. Vaccine Immunol. 2017. [CrossRef] [PubMed]

13. Zhang, J.; Kaiser, M.G.; Deist, M.S.; Gallardo, R.A.; Bunn, D.A.; Kelly, T.R.; Dekkers, J.C.; Zhou, H.; Lamont, S.J. Transcriptome analysis in spleen reveals differential regulation of response to newcastle disease virus in two chicken lines. Sci. Rep. 2018, 8. [CrossRef] [PubMed]

14. Saelao, P.; Wang, Y.; Gallardo, R.A.; Lamont, S.J.; Dekkers, J.M.; Kelly, T.; Zhou, H. Novel insights into the host immune response of chicken Harderian gland tissue during Newcastle disease virus infection and heat treatment. BMC Vet. Res. 2018, 14. [CrossRef] [PubMed]

15. Wang, Y.; Lupiani, B.; Reddy, S.M.; Lamont, S.J.; Zhou, H. RNA-seq analysis revealed novel genes and signaling pathway associated with disease resistance to avian influenza virus infection in chickens. Poult. Sci. 2014, 93, 485-493. [CrossRef] [PubMed]

16. Lakshmanan, N.; Kaiser, M.G.; Lamon, S.J. Marek's disease resistance in MHC-congenic lines from Leghorn and Fayoumi breeds. In Current Research on Marek's Disease, Proceedings of the 5th International Symposium, Kennet Square, PA, USA; American Association of Avian Pathologists: Jacksonville, FL, USA, 23 July 1996.

17. Wang, Y.; Saelao, P.; Chanthavixay, K.; Gallardo, R.; Bunn, D.; Lamont, S.J.; Dekkers, J.M.; Kelly, T.; Zhou, H. Physiological responses to heat stress in two genetically distinct chicken inbred lines. Poult. Sci. 2017, 97, 770-780. [CrossRef] [PubMed]

18. Kültz, D.; Li, J.; Gardell, A.; Sacchi, R. Quantitative molecular phenotyping of gill remodeling in a cichlid fish responding to salinity stress. Mol. Cell. Proteom. 2013, 12, 3962-3975. [CrossRef] [PubMed]

19. Kültz, D.; Li, J.; Paguio, D.; Pham, T.; Eidsaa, M.; Almaas, E. Population-specific renal proteomes of marine and freshwater three-spined sticklebacks. J. Proteom. 2016, 135, 112-131. [CrossRef] [PubMed]

20. Szklarczyk, D.; Morris, J.H.; Cook, H.; Kuhn, M.; Wyder, S.; Simonovic, M.; Santos, A.; Doncheva, N.T.; Roth, A.; Bork, P.; et al. The STRING database in 2017: Quality-controlled protein-protein association networks, made broadly accessible. Nucleic Acids Res. 2017, 45, D362-D368. [CrossRef] [PubMed]

21. R Core Team. R: A Language and Environment for Statistical Computing. R Found Stat Comput Vienna Austria. 2018. Available online: https:/ / www.R-project.org/ (accessed on 20 July 2018).

22. Andrew, S. FastQC: A Quality Control Tool for High Throughput Sequence Data. Available online: http: / / www.bioinformatics.babraham.ac.uk/projects/fastqc (accessed on 20 July 2018).

23. Dobin, A.; Davis, C.A.; Schlesinger, F.; Drenkow, J.; Zaleski, C.; Jha, S.; Batut, P.; Chaisson, M.; Gingeras, T.R. STAR: Ultrafast universal RNA-seq aligner. Bioinformatics 2013, 29, 15-21. [CrossRef] [PubMed]

24. Anders, S.; Pyl, P.T.; Huber, W. HTSeq-A Python framework to work with high-throughput sequencing data. Bioinformatics 2015, 31, 166-169. [CrossRef] [PubMed]

25. Robinson, M.D.; McCarthy, D.J.; Smyth, G.K. edgeR: A Bioconductor package for differential expression analysis of digital gene expression data. Bioinformatics 2010, 26, 139-140. [CrossRef] [PubMed]

26. Krämer, A.; Green, J.; Pollard, J.; Tugendreich, S. Causal analysis approaches in Ingenuity Pathway Analysis. Bioinformatics 2014, 30, 523-530. [CrossRef] [PubMed] 
27. Stray-Pedersen, A.; Backe, P.H.; Sorte, H.S.; Mørkrid, L.; Chokshi, N.Y.; Erichsen, H.C.; Gambin, T.; Elgstøen, K.B.; Bjørås, M.; Wlodarski, M.W.; et al. PGM3 mutations cause a congenital disorder of glycosylation with severe immunodeficiency and skeletal dysplasia. Am. J. Hum. Genet. 2014, 95, 96-107. [CrossRef] [PubMed]

28. Zhang, Y.; Yu, X.; Ichikawa, M.; Lyons, J.J.; Datta, S.; Lamborn, I.T.; Jing, H.; Kim, E.S.; Biancalana, M.; Wolfe, L.A.; et al. Autosomal recessive phosphoglucomutase 3 (PGM3) mutations link glycosylation defects to atopy, immune deficiency, autoimmunity, and neurocognitive impairment. J. Allergy Clin. Immunol. 2014, 133, 1400-1409. [CrossRef] [PubMed]

29. Milner, J.D.; Lyons, J.J.; Zhang, Y.; Yu, X.; Datta, S.; Lamborn, I.T.; Biancalana, M.R.; Wolfe, L.A.; DiMaggio, T.; Matthews, H.F.; et al. Impaired glycosylation due to autosomal recessive PGM3 mutations results in atopy, immune deficiency, autoimmunity, and neurocognitive impairment. J. Allergy Clin. Immunol. 2014, 133, AB161. [CrossRef]

30. Kroeker, A.L.; Ezzati, P.; Halayko, A.J.; Coombs, K.M. Response of primary human airway epithelial cells to influenza infection: A quantitative proteomic study. J. Proteome Res. 2012, 11, 4132-4146. [CrossRef] [PubMed]

31. Herr, N.; Bode, C.; Duerschmied, D. The effects of serotonin in immune cells. Front. Cardiovasc. Med. 2017, 4, 48. [CrossRef] [PubMed]

32. Shabab, T.; Khanabdali, R.; Moghadamtousi, S.Z.; Kadir, H.A.; Mohan, G. Neuroinflammation pathways: A general review. Int. J. Neurosci. 2017, 127, 624-633. [CrossRef] [PubMed]

33. Virella, G.; Lopes-Virella, M.F. The role of the immune system in the pathogenesis of diabetic complications. Front. Endocrinol. 2014, 5, 126. [CrossRef] [PubMed]

34. Edfors, F.; Danielsson, F.; Hallström, B.M.; Käll, L.; Lundberg, E.; Pontén, F.; Forsström, B.; Uhlén, M. Gene-specific correlation of RNA and protein levels in human cells and tissues. Mol. Syst. Biol. 2016, 12, 883. [CrossRef] [PubMed]

35. Liu, Y.; Beyer, A.; Aebersold, R. On the dependency of cellular protein levels on mrna abundance. Cell 2016, 165, 535-550. [CrossRef] [PubMed]

36. Öhrvik, H.; Wittung-Stafshede, P. Identification of new potential interaction partners for human cytoplasmic copper chaperone Atox1: Roles in gene regulation? Int. J. Mol. Sci. 2015, 16, 16728-16739. [CrossRef] [PubMed]

37. Ozato, K.; Shin, D.-M.; Chang, T.-H.; Morse, H.C. TRIM family proteins and their emerging roles in innate immunity. Nat. Rev. Immunol. 2008, 8, 849-860. [CrossRef] [PubMed]

38. Day, A.J. The C-type carbohydrate recognition domain (CRD) superfamily. Biochem. Soc. Trans. 1994, 22, 83-88. [CrossRef]

39. Hogenkamp, A.; van Eijk, M.; van Dijk, A.; van Asten, A.J.A.M.; Veldhuizen, E.J.A.; Haagsman, H.P. Characterization and expression sites of newly identified chicken collectins. Mol. Immunol. 2006, 43, 1604-1616. [CrossRef] [PubMed]

40. McGuinness, O.P. Defective glucose homeostasis during infection. Annu. Rev. Nutr. 2005, 25, 9-35. [CrossRef] [PubMed]

(C) 2018 by the authors. Licensee MDPI, Basel, Switzerland. This article is an open access article distributed under the terms and conditions of the Creative Commons Attribution (CC BY) license (http://creativecommons.org/licenses/by/4.0/). 